\title{
Experimental study of a piezoelectric cantilever beam under droplet impact
}

\author{
Guannan $\mathrm{Hao}^{1}$, Zengliang $\mathrm{Li}^{1}$, Xiangwei Dong ${ }^{1 \mathrm{a}}$ \\ ${ }^{1}$ College of mechanical and electronic engineering, China University of Petroleum (East China), 66 Changjiang Rd, Huangdao District, \\ Qingdao, China 266580
}

\begin{abstract}
The dynamic response of a piezoelectric cantilever beam under droplet impact is investigated by conducting impact tests. Both droplet dynamic behaviours and voltage output can be acquired simultaneously by means of high-speed camera capturing technique. The surface wettability and the macrotextures affect the voltage generation in different ways. For all droplet sizes, both the superhydrophobic-treated and untreated surfaces of the cantilever beam can generate the same magnitude of peak voltage. However, at lower impact velocity, the voltage generated from the untreated surface is slightly higher than the treated surface due to different impact mechanisms upon droplet-substrate interactions. For higher impact velocity, large-scaled droplets can experience splash and water spilling phenomena on the treated and untreated surface respectively, leading to mechanical energy loss of the system. But the treated surface shows a better performance. With the presence of a single macrotexture on the treated surface, there is a critical impact velocity which determines the transition of voltage output. For small-scaled droplets, the surface with the presence of a single macrotexture outperforms only with velocities over the critical value. For larger droplet size, the same trend can be obtained but the effect of the macrotexture is less significant. These outcomes from impact experiments may lay a foundation for future study of exploring new surfaces for piezoelectric energy harvesting devices in the aim of improving the raindrop energy recovery efficiency.
\end{abstract}

\section{Introduction}

In the field of piezoelectric raindrop energy harvesting (PREH) applications, the moderate kinetic energy of raindrops has shown great potential in powering lowenergy electronic devices such as remote sensors and piezo-MEMS by using piezoelectric materials $[1,2]$. The mechanical-to-electrical energy conversion is affected by various factors, such as the performance of piezoelectric materials, the electromechanical coupling efficiency, and the rectifying circuit efficiency, etc. The analogy made between a piezoelectric material and a capacitor allows to estimate the electrical energy collected and the power output [3]. A few of PREH harvesters based on sensor beams have been extensively studied theoretically [4] and experimentally $[5,6]$ in last decades.

When the substrate of a sensor beams is subjected to impacting force given by the water droplet, the voltage generated is associated with the beam deformation which is greatly affected by the beam stiffness. For instance, the peak voltage generated from a harvester is related to the largest deflection of a cantilever beam which is scaled as $\delta_{0} \sim F_{0} L^{\mathrm{g}} / E I$ where $F_{0}$ and $L$ is the maximum impacting force and the beam length respectively, and EI denotes the beam stiffness. It shows that the lower the stiffness of sensor beam, the greater the voltage can be generated hence more energy can be collected. Recent studies showed that compared to the most widely used materials of PZT (e.g., Lead Zirconate Titanate) in many vibration-based energy harvesting devices, the PVDF (e.g., Polyvinylidene fluoride) performs better in harvester utilizations due to its high flexibility, lightweight, and low mechanical impedance. D. Vatansever et al. [7] first demonstrated the ability of PVDF in generating higher voltage output than PZT by performing experiments with various beam dimensions. As the PVDF is more likely to match with the magnitude of raindrop momentum, it is being increasingly used and many available commercial PVDF sensors also provide convenience for conducting impact tests. Besides, different structures of substrate (e.g., cantilever, bridge, floating circles, etc.) have been proposed in order to improve the energy conversion efficiency. After comparison, it was reported [8] that the cantilever configuration is still the best candidate in extracting energy which leads to the greatest beam deformation under the same impact conditions. For cantileverstructured piezoelectric sensors, a prototype made of PZT was developed and tested in both simulated rain and actual rain conditions. Results showed that a total energy of $38.89 \mathrm{uJ}, 52.05 \mathrm{uJ}$ and $114.68 \mathrm{uJ}$ can be collected under light, moderate and heavy rain pattern respectively during five minutes $[9,10]$.

At a physical view upon impact, a droplet can experience general phases of spreading, retraction, bouncing or splash with the increase of the impact

\footnotetext{
a Corresponding author: dongxw139@163.com
} 
velocity. These dynamic behaviours of droplet during droplet-substrate interactions have a nonnegligible effect on the dynamic output of a sensor beam under droplet impact. Since the first model proposed by Guigon et al. [4], the droplet impact is considered as an inelastic collision. This droplet-substrate collision during impact is usually not complete due to the splash phenomenon which is must associated to an impact efficiency $[11,12]$. But few of quantitative studies have been made concerning the efficiency of the impact process. In fact, when droplet splash occurs, a large amount of satellite drops are formed which deliver a proportion of the initial kinetic energy of the impacting droplet, leading to a mechanical loss of the system. Besides, the droplet splash is an important impact mechanism that affects the voltage generation because of the high occurrence of great falling speed of raindrops in actual precipitations. Thus the splash limit of droplet plays a key role in analysing the dynamic response (i.e., output voltage, beam deformation, and droplet dynamics) of the sensor beams. Therefore, understanding the mechanism of droplet impact dynamics is essential to further optimizing the efficiency of piezoelectric raindrop energy harvesters. Considering the flexibility of substrate, the criteria related to splash limit become somewhat inefficient. For instance, the expression developed by Mundo et al. [13] (i.e., $R e^{0.25} W e^{0.5}>57.7$ ) depending on the Weber number ( $\mathrm{We}$ ) and the Reynolds number $(R e)$ is the most widely used in determining the threshold when droplet transits on splash mode. But it was generally used for rigid substrate. Indeed, the splash limit can be altered by many factors, such as the surface wettability, the elasticity and roughness of the substrate, etc. Earlier study suggested that [14, 15] the energy harvesting efficiency can be improved by exploring new surface materials to maximize inelastic collision. In our previous study, a PVDF cantilever harvester was developed and multiple impacts experiments were conducted to model the successive impacts of raindrops in actual condition. Results showed that a superhydrophobic $(\mathrm{SH})$ surface can generate constant and stable voltage output without any effect of the water layer accumulated. Furthermore, the contact time of droplet-substrate interactions can also be greatly reduced. Moreover, the procedure of fabricating new surfaces, such as the SH surfaces, for energy harvesters is specifically advantageous in anti-freezing or anti-wetting utilizations.

It has been demonstrated that a $\mathrm{SH}$ surface can reduce the contact time even below the typical contact time which is scaled with the inertial-capillary timescale $\tau_{0}=\sqrt{\rho R^{3} / \gamma}$ where $\rho, R$ and $\gamma$ is the density, radius and surface tension of water droplet which is independent of the impact velocity [16]. It was reported $[17,18]$ that a morphology on SH surfaces can redistribute the liquid mass and thereby alter the drop hydrodynamics which lead to further contact time reduction. Previous study showed that [18] by adding macrotextures to nonwetting surfaces, the contact time decreases with the impact velocity in a step-like fashion. The fundamental physics of droplet can be altered during interactions with the presence of a single macrotexture, shown as in Fig. 1. The macrotexture can lead to a total rebound of droplet with a lower impact velocity or two global parts of (satellite) drops divided by the macrotexure with a higher impact velocity. The reduced contact time is mainly dominated by the retraction phase of the droplet. It is assumed that the droplet recoils at a constant velocity with the absence of macrotextures, whereas it is changed to a constant accelerated retraction with the presence of macrotextures. Although it has been extensively studied in many industrial applications such as anti-icing [19], ink-jet printing [20] and pesticide spraying [21], its influence on the electrical output from a piezoelectric material has never been reported. The combined effect of the surface wettability and macrotextures upon droplet-substrate interactions are still unclear. In this study, we focus on the dynamic outputs of a PVDF cantilever harvester by conducting droplet impact experiments. To model the real rainfall conditions, artificial raindrops are formed with typical raindrop sizes of $1.0 \mathrm{~mm} \sim 5.0 \mathrm{~mm}$ [22] by the use of a micropump.

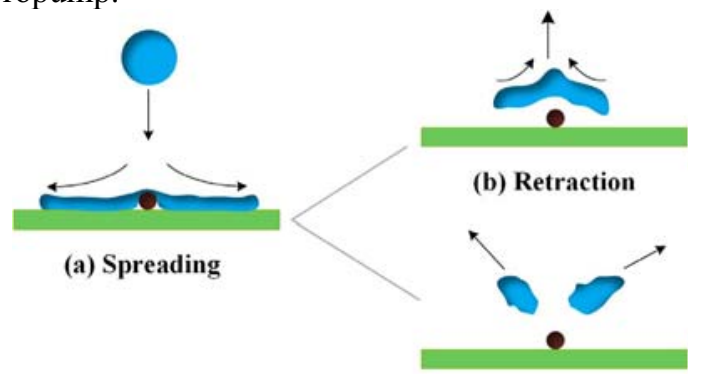

(c) Splitting

Figure 1. Droplet dynamic behaviours altered by a single macrotexture upon substrate

\section{Impact experiments}

For impact experiments, a commercial unimorph PVDF sensor (LDT1-028K) is used. A thin film of PVDF polymers is sandwiched between silver ink and protected by exterior layers of Mylar. It is fixed at a base in a cantilever style and the beam dimension is $41.0 \mathrm{~mm}$ (length) $\times 16.0 \mathrm{~mm}$ (width) $\times 0.2 \mathrm{~mm}$ (thickness), shown as in Fig. 2. A single macrotexture is designed with a nickel wire of radius $b(200 \mu \mathrm{m})$ which is placed along the beam substrate at a distance of $5.0 \mathrm{~mm}$ from the tip end. Both the surface and the wire were treated using a commercial spray with super-hydrophobic nanoparticles to achieve the uniform micro-scaled textures of the surface. By comparison, the untreated beam surface is hydrophilic on which the droplet is supposed to be deposited after the impact. To access the wettability, the apparent advancing and receding contact angles (i.e., $\theta_{a}$ and $\theta_{r}$ ) are measured by the stationary droplet method for both surfaces. The measured values for the treated (i.e., super-hydrophobic) surface are $\theta_{a}=155^{\circ} \pm 1.2^{\circ}$ and $\theta_{r}=148^{\circ} \pm 2.0^{\circ}$, and those for the untreated (i.e., hydrophilic) surface are $\theta_{a}=$ $91.3^{\circ} \pm 3.2^{\circ}$ and $\theta_{r}=49^{\circ} \pm 2.6^{\circ}$, shown as in Fig. 3. 


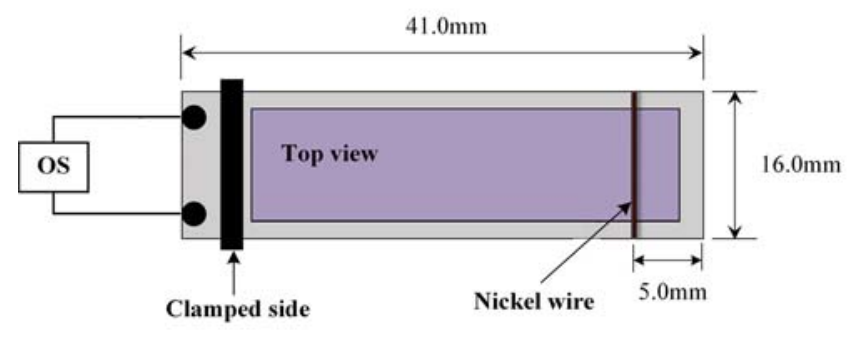

Figure 2. Configuration of the PVDF sensor beam with presence of a nickel wire
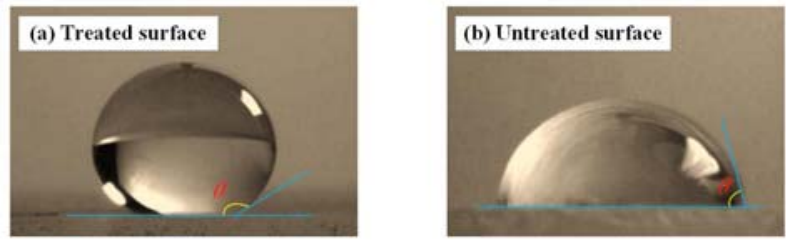

Figure 3. Contact angle illustration of water droplet upon (a) the treated surface and (b) the untreated surface, showing a

super-hydrophobic and hydrophilic feature respectively

Water droplets of diameter $1.5 \mathrm{~mm}, 2.4 \mathrm{~mm}$ and $4.6 \mathrm{~mm}$ are formed from capillary and blunt needles respectively which is supplied by a micropump. Droplets are released at different heights to achieve various impact velocities. A high-speed camera (NAC Company, HX-7 s, Japan) is used to capture the fundamental physics of droplet-substrate interactions at a speed of 10000 frames/second. The actual impact velocity is obtained by measuring the captured images. Combined with a digital oscilloscope, both the dynamic behaviours of the droplet and the beam as well the electrical output can be recorded simultaneously. The voltage is measured in an open-circuit condition with a pure resistance of $100 \mathrm{M} \Omega$. Each impact test is repeated at least three times to validate the reproducibility of impact parameters. To study the comprehensive effect of surface wettability and the macrotexture on the dynamic response of the cantilever beam, two trials of impact tests were considered:

- The surface wettability effect on droplet dynamic behaviours can be studied by using a large-scaled droplet (i.e., $4.6 \mathrm{~mm}$ ) for both treated and untreated surfaces;

- Considering the macrotexture effect, small-scaled and intermediate droplets (i.e., $1.5 \mathrm{~mm}$ and $2.4 \mathrm{~mm}$ ) were used to impinge the treated beam surface at the central section of the nickel wire with various impact velocities.

\section{Results and discussion}

\subsection{Effect of surface wettability}

Water droplets are formed and released at very different heights to achieve a range of impact velocity from $0.9 \mathrm{~m} / \mathrm{s}$ to $3.4 \mathrm{~m} / \mathrm{s}$. For all impact parameters, there is We $>1$ which represents the dominance of droplet inertia compared to the surface tension force during an impact process. Besides, the Capillary number $(\mathrm{Ca}<<1)$ and Ohnesorge number $(O h<<1)$ shows that the capillary effect can be neglected in the scope of this study. It is assumed that all the impacts are in the regime of large-deformation, so that the contact time reduction would be less affected by the distinct droplet dynamics when varying impact velocities when a single macrotexture is considered. It is known that the voltage output follows well the beam deformation in an opencircuit condition, thus the electromechanical behaviours of a piezoelectric harvester can be characterized by two general aspects: droplet dynamics upon droplet-substrate interactions which can affect the beam vibration or deformation, and the voltage generated across the PVDF layer within the beam sensor.

\subsubsection{Effect of droplet dynamic behaviours}

Figure 4 shows the voltage waveform obtained from both the treated and untreated surfaces by using a droplet of $D=4.6 \mathrm{~mm}$. The largest deformation is related to the maximum spreading diameter of the impacting droplet. According to a criterion defined to access the dominance between surface tension and viscosity, the maximum diameter scales as $D_{\max } \sim D \cdot W e^{1 / 4}$ [18], showing that $D_{\max }$ increases with the Weber number $W e$ so as the impact velocity V. As shown in Fig. 4 (a), for a lower impact velocity $V=1.3 \mathrm{~m} / \mathrm{s}$, the peak voltage is almost the same for both surfaces, indicating that the $D_{\max }$ is not affected by the surface wettability. But the total energy collected from the treated surface is supposed to be slightly lower than that of the untreated surface. Figure 4 (b) shows the snapshots of typical instants during the impact. In fact, the water droplet adheres on and vibrates together with the untreated surface after the impact, whereas for the treated surface, the droplet recoils and lifts off from the surface. It is noted that there is a secondary impact for the later concerning the lifted droplet re-impinging the surface with a lower impact velocity than $V$. Although the re-impingement of droplet contributes to a little increase of the total energy recovery, it is not the dominant impact mechanism in the field of PREH and we focus on single droplet impact.

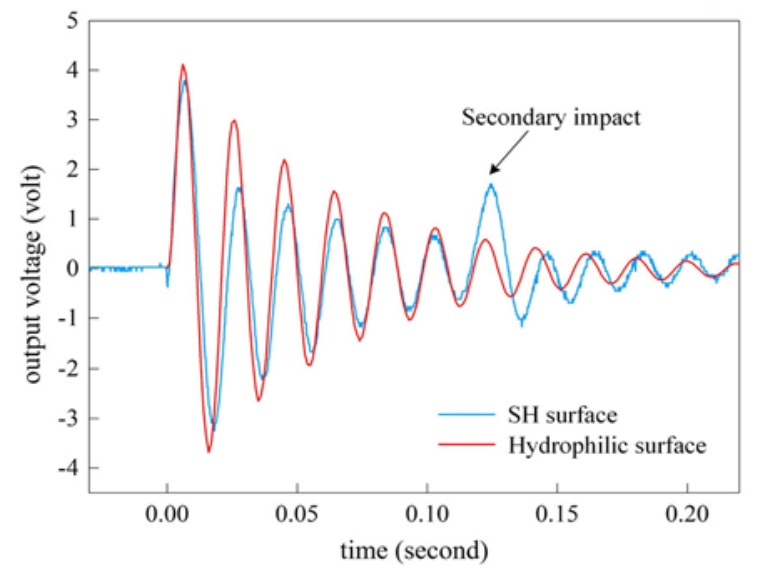

(a) 


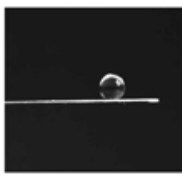

$t=0 \mathrm{~s}$

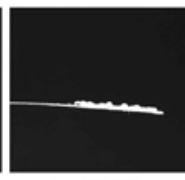

$t=0.006 \mathrm{~s}$

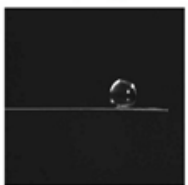

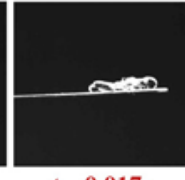

$t=0.017 \mathrm{~s}$

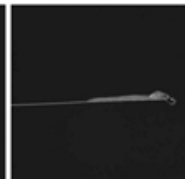

(b)

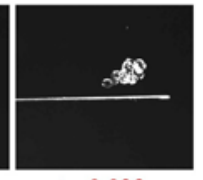

$t=0.023 \mathrm{~s}$

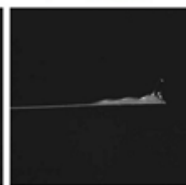

Figure 4. Dynamic output of the PVDF cantilever beam by using the droplet $D=4.6 \mathrm{~mm}$ with a lower impact velocity $V=$ $1.3 \mathrm{~m} / \mathrm{s}$. Fig. 4 (a) illustrates the voltage waveforms for both surfaces, and Fig. 4(b) shows typical snapshots captured with the upper row showing the treated surface and the below row showing the untreated surface

\subsubsection{Water spilling phenomenon}

Figure 5 shows the outcomes by using the same droplet with a higher impact velocity $V=3.35 \mathrm{~m} / \mathrm{s}$. It can be seen that both surfaces generate the same magnitude of peak voltage following which the untreated surface shows a weakened voltage output. It is caused by the water spilling phenomenon during the spreading phase of droplet which can be observed in Fig. 5 (b). For largescaled droplets (e.g., $4.6 \mathrm{~mm}$ ), the $D_{\max }$ exceeds the size limit of the beam and the droplet spills off the surface from side edges, causing a mechanical energy loss of the system. As suggested by Guigon et al. [4], the piezoelectric material should be very thin, not prestressed and with a width slightly smaller than the maximum diameter of the impacting drop. Results show that the water spilling phenomenon can be avoided by the use of water repellent surfaces. In this case, splash phenomenon can be triggered for higher impact velocity with the formation of satellite drops. Nevertheless, the free vibration of the beam after the $D_{\max }$ is less affected by the energy delivered by the satellite drops compared to the scenario with water spilling phenomenon. Overall, the surface wettability affects the voltage output in different ways as the impact velocity increases for largescaled droplets. The threshold of the impact velocity depends on both the droplet size and the beam dimensions.

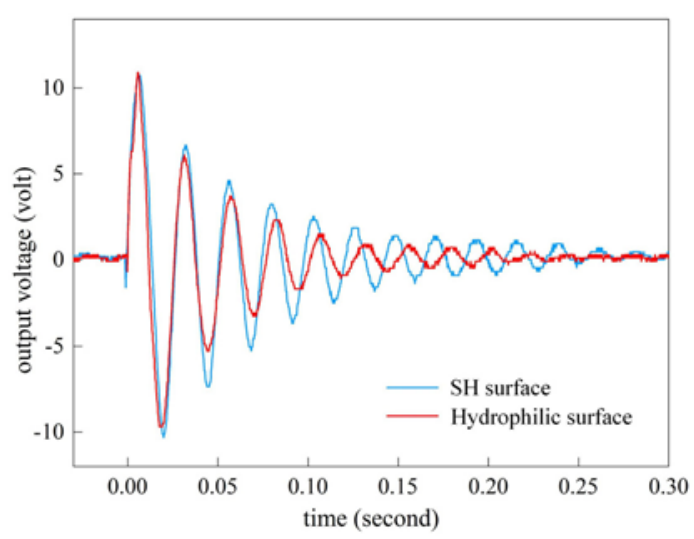

(a)
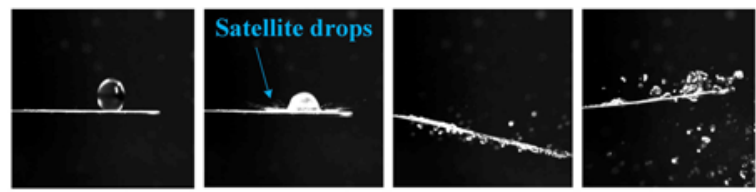

$t=0 \mathrm{~s}$

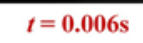

$t=0.017 \mathrm{~s}$

$t=0.023 \mathrm{~s}$
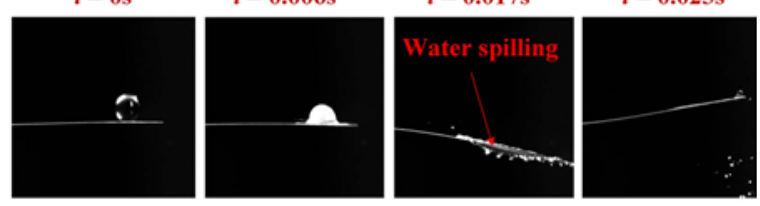

(b)

Figure 5. Dynamic output of the PVDF cantilever beam by using the droplet $D=4.6 \mathrm{~mm}$ with a higher impact velocity $V=$ $3.35 \mathrm{~m} / \mathrm{s}$. Fig. 5 (a) illustrates the voltage waveforms for both surfaces, and Fig. 5(b) shows typical snapshots captured with the upper row showing the severe splash phenomenon of droplet occurred on the treated surface and the below row showing the water spilling phenomenon of droplet occurred on the untreated surface

\subsection{Effect of macrotextures}

Discrete values can be obtained by adding a single macrotexture (i.e., nickel wire) of radius $b=200 \mu \mathrm{m}$ on the treated surface. Figure 6 compares the voltage generated from beam surfaces with and without the presence of the macrotexture by varying impact velocities. A small-scaled droplet of $1.5 \mathrm{~mm}$ is used the spreading diameter of which is comparable with the macrotexture size. Results show that at lower $V$, the macrotexture decreases the voltage output, whereas for higher $V$, it outperforms the merely treated surface, showing a smaller damping ratio. The transition is determined by a critical value of $V=1.32 \mathrm{~m} / \mathrm{s}$. It is associated with the droplet dynamic behaviours altered by the macrotexture. Figure 7 shows typical snapshots for the instant of $0.0036 \mathrm{~s}$ related to the retraction phase of droplet. When the droplet is characterized by the spherical rebound, pancake-shaped rebound and splash with the increasing $V$, the macrotexture has an effect in reducing the contact time and contact area upon dropletsubstrate interactions. With a single macrotexture, the droplet can be divided into two general proportions. Such that, the inelastic collision is maximized which can lead to a higher electrical output. Besides, it was 
reported that a hydrophilic beam surface encourages the accumulation of water upon the energy harvesting harvesters which leads to excessive damping and loss of electrical energy output compared to the hydrophobictreated surfaces [23]. It can be seen that for higher $V$ (i.e., $2.48 \mathrm{~m} / \mathrm{s}$ ), the effect of splash can be enhanced by reducing the contact area, leading to an increased voltage output. By contrast, the voltage can be slightly decreased with lower impact velocities (i.e., $V<1.32 \mathrm{~m} / \mathrm{s}$ ).

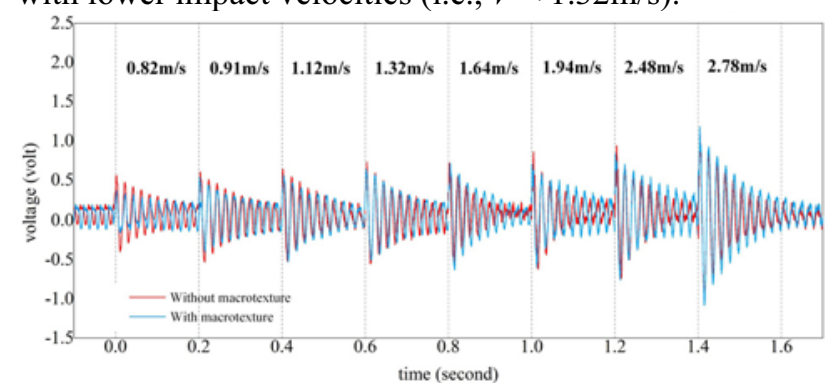

Figure 6. Voltage comparison between surfaces with and without the presence of a single macrotexture by using a droplet of $D=1.5 \mathrm{~mm}$. The impact velocity is varied from $0.82 \mathrm{~m} / \mathrm{s}$ to $2.78 \mathrm{~m} / \mathrm{s}$ wherein a transition of voltage generation can be observed at $V=1.32 \mathrm{~m} / \mathrm{s}$

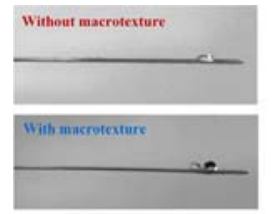

(a) $V=0.82 \mathrm{~m} / \mathrm{s}$

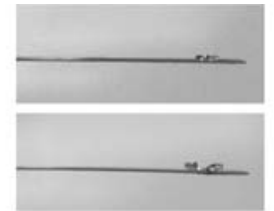

(d) $V=1.32 \mathrm{~m} / \mathrm{s}$

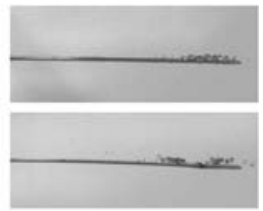

(g) $V=2.48 \mathrm{~m} / \mathrm{s}$
Figure 7. Macrotexture effect on regime transition of droplet dynamics with all snapshots captured at $0.0036 \mathrm{~s}$

By comparison, a larger droplet of $2.4 \mathrm{~mm}$ is tested under the same impact parameters. As shown in Fig. 8, the same trend can be obtained, but the difference of voltage is less significant compared to the case of droplet $D=1.5 \mathrm{~mm}$. Nevertheless, the critical impact velocity for the voltage transition is the same (i.e., $1.32 \mathrm{~m} / \mathrm{s}$ ). It shows the independence of the critical impact velocity to the droplet size. It is noted that for lower $V$ (i.e., $0.82 \mathrm{~m} / \mathrm{s}$ ) shown as in Fig. 9, droplet dynamics are greatly changed by the macrotexture. After the maximum spreading at $0.003 \mathrm{~s}$, the retraction of droplet is delayed by the macrotexture. However, it has little influence on the voltage output. Because for intermediate droplet sizes such as $2.4 \mathrm{~mm}$, the droplet inertia is dominant in droplet-substrate interactions hence the voltage generated. Thus the effect of macrotexture is less significant for larger droplet sizes.

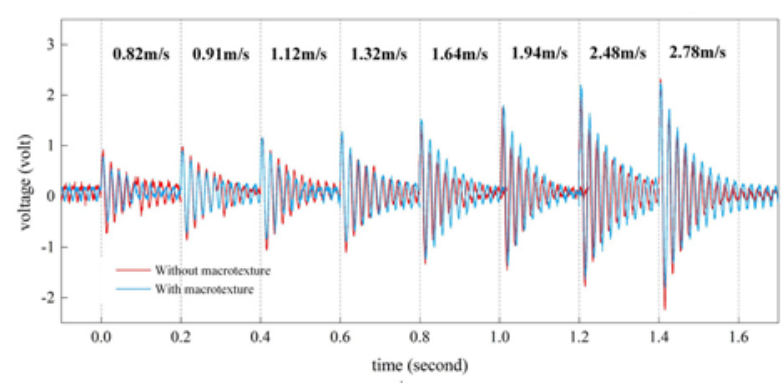

Figure 8. Voltage comparison between surfaces with and without the presence of a single macrotexture by using a droplet of $D=2.4 \mathrm{~mm}$. The impact velocity is varied from $0.82 \mathrm{~m} / \mathrm{s}$ to $2.78 \mathrm{~m} / \mathrm{s}$ wherein a transition of voltage generation can be observed at $V=1.32 \mathrm{~m} / \mathrm{s}$

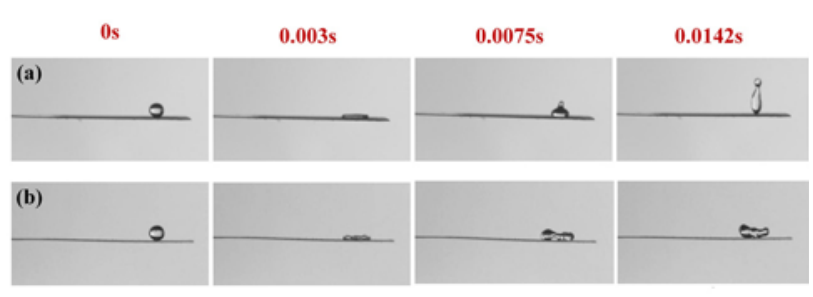

Figure 9. Droplet dynamics of a droplet $D=2.4 \mathrm{~mm}$ with a lower impact velocity $V=0.82 \mathrm{~m} / \mathrm{s}$ on the treated surfaces (a) without and (b) with a single macrotexture

\section{Conclusion}

The effect of surface property on the dynamic outputs (i.e., droplet dynamics and voltage output) of a piezoelectric cantilever under droplet impact is investigated. Experimental outcomes show that the SHtreated surfaces perform better for large-scaled droplets with higher impact velocities. The water spilling phenomenon occurred during droplet spreading phase on the untreated beam surfaces leads to a mechanical energy loss of the system which weakens the voltage output. Considering the macrotexture effect, a single nickel wire of radius $200 \mu \mathrm{m}$ is used which shows more significant effect for small-scaled droplets (e.g., 1.5mm) than for larger droplet size (e.g., $2.4 \mathrm{~mm}$ ). With the presence of macrotextures, the retraction of droplet can be greatly changed, leading to a reduced contact area upon dropletsubstrate interactions. A critical value of impact velocity is determined which is independent of the droplet size. Overall, the surface wettability combined with macrotextures can lead to discrete values on voltage output depending on various parameters (e.g., impact velocity and droplet size). In the field of PREH, the terminal velocity of raindrops is usually important, thus more energy can be collected when a super-hydrophobic surface combined with a single macrotexture is applied for droplet size which is comparable with the macrotexture size. It provides a foundation to further study the enhancement of substrate surface property and the electrical energy harvested toward PREH applications. 


\section{References}

1. G. Zhou and L. Huang and W. Li, et al., Journal of Sensors 2014, 1 (2014).

2. C. Wong and Z. Dahari and A. Abd Manaf, et al., Journal of Electronic Materials 44, 13 (2015).

3. F. Viola and P. Romano and R. Miceli, et al., (IEEE, 2014), p. 1.

4. R. Guigon and J. Chaillout and T. Jager, et al., Smart Materials and Structures 17, 15038 (2008).

5. R. Guigon and J. Chaillout and T. Jager, et al., Smart Materials and Structures 17, 15039 (2008).

6. V. K. Wong and J. H. Ho and E. H. Yap, Advanced Materials Research 1043, 263 (2014).

7. D. Vatansever and R. L. Hadimani and T. Shah, et al., Smart Materials and Structures 20, 55019 (2011).

8. F. Viola, Applied Sciences 8, 955 (2018).

9. Z.Z. Ong, V.K. Wong and J.H. Ho. Sensors and Actuators A: Physical 252, 154-164 (2016).

10. V.K. Wong, J.H. Ho and A.B. Chai. Energy 124, 364-371 (2017).

11. K. G. CHUA and Y. F. HOR and H. C. LIM, Sensors \& transducers 200, 1 (2016).

12. K. C. R. Perera et al. International Journal of Energy and Power Engineering 8(2), 325-330 (2014).

13. C. Mundo and M. Sommerfeld and C. Tropea, International Journal of Multiphase Flow 21, 151 (1995).

14. M. A. Ilyas and J. Swingler, Energy 90, 796 (2015).

15. G. Hao and X. Dong and Z. Li, et al., Journal of adhesion science and technology, 1 (2020).

16. A. Raiyan and T. S. Mclaughlin and R. K. Annavarapu, et al., Scientific Reports 8 (2018).

17. J. C. Bird and R. Dhiman and H. Kwon, et al., Nature 503, 385 (2013).

18. A. Gauthier and S. Symon and C. Clanet, et al., Nature Communications 6 (2015).

19. T. Vasileiou and T. M. Schutzius and D. Poulikakos, Langmuir 33, 6708 (2017).

20. D. B. van Dam and C. Le Clerc, Physics of Fluids 16, 3403 (2004).

21. D. B. Smith et al., Trans. ASAE 43, 255 (2000)

22. P.V. Biswas, M.A. Uddin, M.A. Islam and et al. International conference on mechanical engineering 2009.

23. S. C. J. Jellard and S. H. Pu and S. Chen, et al., Smart materials and structures 28, 95002 (2019). 